\title{
Supravalvular aortic stenosis
}

INSERM

\section{Source}

INSERM. (1999). Orphanet: an online rare disease and orphan drug data base.

Supravalvular aortic stenosis. ORPHA:3193

SupraValvar Aortic Stenosis (SVAS) is characterized by the narrowing of the aorta lumen (close to its origin) or other arteries (branch pulmonary arteries, coronary arteries). This narrowing of the aorta or pulmonary branches may impede blood flow, resulting in heart murmur and ventricular hypertrophy (in case of aorta involvement). The narrowing results from a thickening of the artery wall, which is not related to atherosclerosis. 